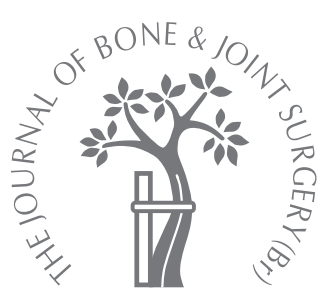
W. Y. Kim,
C. E. Hutchinson,
J. G. Andrew,
P. D. Allen

From Salford Royal Hospitals, NHS

Trust, Salford, England

\title{
The relationship between acetabular retroversion and osteoarthritis of the hip
}

\begin{abstract}
Excessive acetabular cover secondary to a retroverted acetabulum causes pincer impingement, which may cause early osteoarthritis of the hip. Our aim was to determine if there was a relationship between acetabular version and osteoarthritis of the hip. Using image processing and analysis software we studied $117 \mathrm{CT}$ images of the hip in patients aged less than 65 years who had undergone a CT virtual colonoscopy. The mean CT joint space of the 18 hips with acetabular retroversion was narrower compared with the 99 hips with normal acetabular alignment $(p<0.0001)$. A correlation of $r=0.46(p<0.01)$ was found between right hip acetabular version and the mean right hip joint space and of $r=0.31(p=0.02)$ between left hip acetabular version and the mean left hip joint space. Acetabular retroversion is associated with radiological evidence of osteoarthritis of the hip. An understanding of the mechanical basis of osteoarthritis of the hip allows early treatment of the underlying structural abnormality and prevents progression of the degenerative condition.
\end{abstract}

The aetiology of primary osteoarthritis of the hip is not fully understood. It has been suggested that it may be attributable to unrecognised or mild hip dysplasia, previous slipped capital femoral epiphysis, Perthes' disease, or other morphological abnormalities around the hip. ${ }^{1-3}$ Femoroacetabular impingement has also been recognised recently as a cause of early osteoarthritis of the hip. ${ }^{4}$ Excessive acetabular cover secondary to a retroverted acetabulum causes pincer impingement, which may be a mechanism by which early osteoarthritis of the hip develops.

Version of the acetabulum refers to alignment in the sagittal plane. In non-dysplastic hips, a retroverted acetabulum represents an abnormality of orientation of an otherwise normal acetabulum. In dysplastic hips, a retroverted acetabulum is one in which the posterior portion of the acetabulum is deficient.

Several studies have confirmed that acetabular joint reaction forces are directed posteriorly and superiorly, when viewed in the sagittal planes. ${ }^{5,6}$ The highest acetabular contact pressures occur in its posterior aspect. ${ }^{7}$ Any deficiency of the posterior wall, due to a malorientation or dysplasia, will therefore result in increased contact stresses, which possibly contribute to the development of osteoarthritis of the hip.

Our aims were to determine the prevalence of acetabular retroversion and to examine whether there was an association between radiological evidence of osteoarthritis of the hip and a retroverted acetabulum.

\section{Patients and Methods}

We used CT virtual colonoscopy images performed in the radiology department of our institution (Salford Royal Hospitals NHS Trust). This is the investigation of choice for pathology of the bowel and incidentally includes both hips. Images of the hip in patients aged less than 65 years were downloaded onto a personal computer (Dell Inspiron 9300, Bracknell, United Kingdom). No patient was known to have osteoarthritis of the hip. These images were analysed using an image-processing and analysis software package (ImageJ, free software: http://rsb. info.nih.gov/ij/index.html). We obtained ethical approval.

A total of 125 hips were reviewed, although eight were excluded, seven because the images were poor and one because of an excessively prominent osteophyte which made assessment of acetabular version unreliable. We therefore analysed a total of 117 hips (56 left and 61 right). There were 52 men and 65 women, with a mean age of 51.9 years (20 to 64 ).

Routine scanning parameters were used for CT virtual colonoscopy. These consisted of approximately 300 images of axial helical scans using CT (four-slice CT scanner, IGE 
Table I. CT grading of acetabular version according to Tonnis and Heinecke ${ }^{10}$

\begin{tabular}{lll}
\hline Grade of version & Degrees & Description \\
\hline+3 & $>25$ & Severely increased \\
+2 & 21 to 25 & Moderately increased \\
+1 & 15 to 20 & Assumed normal range \\
-2 & 10 to 14 & Moderately decreased \\
-3 & $<10$ & Severely decreased \\
\hline
\end{tabular}

Table II. Protocol for the measurement of the hip joint space (ImageJ software)

Crop image

Select Image

Stacks

Reslice

Input Z spacing $1 \mathrm{~mm}$

Crop image

Enhance contrast $0.5 \%$ saturated pixels

Identify floor of acetabulum

Magnify $(300 \%)$

Measure acetabular joint space $(\mathrm{mm})$

Pace; IGE Medical Systems Ltd, Slough, United Kingdom, scan speed $0.6 \mathrm{~s}$, axial thickness $2.5 \mathrm{~mm}$ with $1.25 \mathrm{~mm}$ overlapping intervals).

Each hip was scanned proximal to the articular surface, progressing to the distal aspect of the acetabulum. The level of the superior third of the hip in the sagittal plane was identified. The sagittal CT images of the superior one-third of an acetabulum were examined for version, as suggested by $\mathrm{Li}$ and Ganz. ${ }^{8}$ The acetabular version was corrected for pelvic tilt in the coronal plane, if tilt was identified. All the hips were congruent, at the site of measurement of version, with prone images being chosen to minimise the effect of pelvic tilt. ${ }^{9}$ This method of measurement of acetabular version has been previously described and was classified according to the criteria of Tonnis and Heinecke ${ }^{10}$ (Table I).

Osteoarthritis of the hip was assessed by the mean joint space in the superior and lateral aspects of the acetabulum. CT coronal reconstruction images were used to identify the superolateral aspect and floor of the acetabulum in order to standardise the site of measurement of the mean joint space. This was measured three times, and in separate areas in the superior aspect of the acetabulum, in order to obtain a mean value. Ossification of the rim of the acetabulum was noted, if present. A uniform protocol for image enhancement, reconstruction and measurement of the joint space was used throughout the study (Table II).

Reproducibility. Intra-observer reproducibility of the measurements of joint space and version was assessed by blinded re-reading of a subset of 50 hips, six weeks after the first reading, and using the method of Bland and Altman. ${ }^{11}$ Statistical analysis. The difference in the mean joint space between the retroverted and normally aligned acetabula was investigated with a two-tailed test. The relationship between acetabular version and osteoarthritis of the hip was investigated by Pearson's correlation coefficient test. We considered $\mathrm{p}<0.05$ to be significant.

\section{Results}

The prevalence of acetabular retroversion was $15.4 \%$ (18 hips) according to the criteria of Tonnis and Heinecke. ${ }^{10}$ The values of version and mean joint space followed a normal distribution. The mean CT joint space of the 18 hips with acetabular retroversion was narrower compared with the 99 hips with normal acetabular alignment $(1.60 \mathrm{~mm} v \mathrm{~s}$ $2.35 \mathrm{~mm})$ which was statistically significant $(\mathrm{p}<0.0001)$. The mean $(95 \% \mathrm{CI})$ acetabular version of the right hip was $24.4^{\circ}\left(21.7^{\circ}\right.$ to $\left.27.0^{\circ}\right)$ and the mean right hip joint space $2.20 \mathrm{~mm}$ (2.08 to 2.32). A correlation coefficient (95\% CI) of $\mathrm{r}=0.46(0.23$ to $0.64 ; \mathrm{p}<0.01)$ was found between right hip acetabular version and the mean right joint space. The mean $(95 \% \mathrm{CI})$ acetabular version of the left hip was $25.8^{\circ}$ $\left(23.4^{\circ}\right.$ to $\left.28.1^{\circ}\right)$ and the mean left joint space $(95 \%$ CI $) 2.29$ $\mathrm{mm}(2.18$ to 2.41$)$. A correlation coefficient $(95 \% \mathrm{CI})$ of $\mathrm{r}=0.31(0.05$ to $0.53 ; \mathrm{p}=0.02)$ was found between left hip acetabular version and the mean left joint space. Ossification of the acetabular rim was noted in four hips, all of which had grade- 3 acetabular version according to the criteria of Tonnis and Heinecke. ${ }^{10}$

Intra-observer reproducibility (95\% limits of agreement) was acceptable for acetabular version measurements and joint space to within $3.43^{\circ}\left(-2.02^{\circ}\right.$ to $\left.+3.43^{\circ}\right)$ and $0.21 \mathrm{~mm}$ $(-0.18$ to +0.21$)$.

\section{Discussion}

Most cases of osteoarthritis of the hip can be attributed to an underlying aetiology, including mild or unrecognised hip dysplasia and previous trauma, Perthes' disease or slipped upper femoral epiphysis. ${ }^{1-3}$ Femoroacetabular impingement has also been identified as a cause of early osteoarthritis of the hip. There are two types of femoroacetabular impingement; cam and pincer. The former occurs because of an abnormal shape of the head-neck junction of the femur, as in a 'bullet-shaped' femoral head or slipped upper femoral epiphysis. Excessive acetabular cover secondary to a retroverted acetabulum causes pincer impingement. Both forms may lead to early osteoarthritis of the hip., ${ }^{4,12}$

Passive movement of the hip into simultaneous flexion, adduction and internal rotation, especially in the mid-range of flexion, is a provocative sign of impingement. ${ }^{13}$ There may be an associated acetabular labral tear. Degeneration of the labrum with fragmentation and ossification of the superior rim of the acetabulum follows repeated abutment of the acetabular rim and the femoral head-neck junction. This can result in a chondral injury to the posteroinferior acetabulum. ${ }^{14}$ Ossification of the rim of the acetabulum was clearly noted in four hips in our series. All four had grade- 3 retroverted acetabula.

Our results are comparable with those of recent studies, which suggest an association between acetabular retroversion and osteoarthritis of the hip. ${ }^{15,16}$ These studies used 
pelvic radiographs, identified retroverted acetabula through the 'cross-over' sign and correlated retroverted acetabula with radiological evidence of osteoarthritis of the hip. The cross-over sign is seen when the anterior rim of the acetabulum 'crosses over' its posterior rim, as their outlines are traced from proximal to distal on the radiograph. ${ }^{15}$ In addition, the outline of the edge of the posterior wall of a retroverted acetabulum passes medial to the centre of the femoral head in a retroverted acetabulum, compared with through or lateral to the femoral head in the normal or anteverted acetabulum. ${ }^{15}$ However, it is not possible to quantify the degree of version of the acetabulum using radiographs. Furthermore, pelvic tilt in the coronal and sagittal planes alters the appearance of acetabular version and must be accounted for in its assessment. ${ }^{12}$ Prone images were analysed in our series in order to obtain a neutral position of the pelvis.

CT provides greater anatomical detail while allowing correction for the presence of tilt in the coronal plane. Previous CT studies of acetabular version may have underestimated true version since it is necessary to scan the superior third of the acetabulum in order to avoid missing retroversion. ${ }^{8,12}$ The prevalence of retroversion in our study was $15.4 \%$, which is comparable with values found in the literature of between $5 \%$ and $20 \% .^{8,16}$

There are several limitations to our study. Radiological evidence of osteoarthritis of the hip may not correlate with symptoms. The assessment of the severity of the condition may be subjective and prone to inter- and intra-observer variation if plain radiographs are used. Joint space has been shown to correlate most closely with the symptoms of osteoarthritis compared with the presence of osteophytes, cysts or subchondral sclerosis. ${ }^{17}$ The parameter is objective, being easily and reproducibly measured using CT. Patients of a relatively young age $(<65$ years) were included in the study since the older age groups might have had excessive narrowing of the joint space or the presence of osteophytes, making the assessment of acetabular version and the joint space both difficult and unreliable. The relatively young age of our patients (mean 51.9 years; 20 to 64) may account for the absence of an inverse correlation between age and joint space.

Our study did not assess the effect of femoral version in patients with decreased acetabular version; this may have a compensatory effect on a retroverted acetabulum. ${ }^{10}$ It may in part explain why some patients with reduced acetabular version do not develop early osteoarthritis of the hip. Furthermore, we did not study the possibility of cam impingement or abnormalities of the head-neck junction of the femur, since CT virtual colonoscopy images do not extend far enough down the femur to allow this analysis. The available CT images allowed accurate measurement of acetabular version and the mean joint space. Further work may involve the use of MRI to assess the volume of the articular cartilage, which may be a more accurate indicator of the loss of articular cartilage and osteoarthritis of the hip.

Our results provide evidence that acetabular retroversion contributes to the development of osteoarthritis of the hip. In symptomatic patients, surgery to address the underlying structural abnormality is indicated. Advances in arthroscopic methods may allow treatment of mild but symptomatic acetabular retroversion. It is important, with the increasing use of arthroscopy for the treatment of labral tears, that an underlying diagnosis of acetabular retroversion should be considered. In severe cases of acetabular retroversion, peri-acetabular osteotomy may be indicated.

No benefits in any form have been received or will be received from a commercial party related directly or indirectly to the subject of this article.

\section{References}

1. Harris WH. Etiology of osteoarthritis of the hip. Clin Orthop 1986;213:20-33.

2. Goodman DA, Feighan JE, Smith AD, et al. Subclinical slipped capital femoral epiphysis: relationship to osteoarthritis of the hip. J Bone Joint Surg [Am] 1997;79-A: 1489-97.

3. Solomon L. Patterns of osteoarthritis of the hip. J Bone Joint Surg [Br] 1976;58-B: 176-83.

4. Beck M, Kalhor M, Leunig M, Ganz R. Hip morphology influences the pattern of damage to the acetabular cartilage: femoroacetabular impingement as a cause of early osteoarthritis of the hip. J Bone Joint Surg [Br] 2005;87-B:1012-18.

5. Pedersen DR, Brand RA, Davy DT. Pelvic muscle and acetabular contact forces during gait. J Biomech 1997;30:959-65

6. Witte H, Eckstein F, Recknagel S. A calculation of the forces acting on the human acetabulum during walking: based on in vivo force measurements, kinematic analysis and morphometry. Acta Anat (Basal) 1997;160:269-80.

7. Hodge WA, Carlson KL, Fijian RS, et al. Contact pressures from an instrumented hip endoprosthesis. J Bone Joint Surg [Am] 1989;71-A:1378-86.

8. Li PLS, Ganz R. Morphologic features of congenital acetabular dysplasia: one in six is retroverted. Clin Orthop 2003:416:245-53.

9. Visser JD, Jonkers A, Hillen B. Hip joint measurement with computerized tomography. J Pediatr Orthop 1982;2:143-6.

10. Tonnis D, Heinecke A. Acetabular and femoral anteversion: relationship with osteoarthritis of the hip. J Bone Joint Surg [Am] 1999;81-A:1747-70.

11. Bland J, Altman DG. Statistical methods for assessing agreement between two methods of clinical measurement. Lancet 1986;1:307-10.

12. Siebenrock KA, Kalbermatten DF, Ganz R. Effect of pelvic tilt on acetabular retroversion: a study of pelves from cadavers. Clin Orthop 2003;407:241-8.

13. Klaue K, Durnin CW, Ganz R. The acetabular rim syndrome: a clinical presentation of dysplasia of the hip. J Bone Joint Surg [Br] 1991;73-B:423-9.

14. Ganz R, Parvizi J, Beck M, et al. Femoroacetabular impingement: a cause for osteoarthritis of the hip. Clin Orthop 2003;417:112-20.

15. Reynolds D, Lucas J, Klaue K. Retroversion of the acetabulum: a cause of hip pain. J Bone Joint Surg [Br] 1999;81-B:281-8.

16. Giori NJ, Trousdale RT. Acetabular retroversion is associated with osteoarthritis of the hip. Clin Orthop 2003;417:263-9.

17. Croft P, Cooper C, Wickham C, Coggon D. Defining osteoarthritis of the hip for epidemiological studies. Am J Epidemiol 1990;132:514-22. 